\title{
The anatomy of the optic chiasma and heteronymous hemianopia
}

\author{
J. E. A. O’CONNELL
}

From the Department of Neurological Surgery, St. Bartholomew's Hospital, London

SUMMARY The gross anatomy of the optic nerves and chiasma has been studied, and differences in the tension in the crossed and uncrossed fibres after chiasmal displacement have been investigated. The anterior and posterior attachments of the medial and lateral fibres of the nerves have been studied. The chiasma has been dissected under low power microscopy and a three dimensional picture of it developed. Bitemporal hemianopia, as well as associated or independent hemianopic scotomata, results from stretching of the crossing fibres in the chiasma. Binasal hemianopia results from compression of the uncrossed fibres in the optic nerve or chiasma by the anterior cerebral or internal carotid arteries. The compression is effective because it is sharply localized and, probably as a result of pulsation, deeply grooves the nerve with a resulting acute distortion of fibres; it is likely that the lax lateral fibres would be less affected by a more widely spread compression. When this defect develops on top of an existing bitemporal hemianopia, it is believed that its usual cause remains the same. The crossed and uncrossed fibres of the optic chiasma differ not only anatomically in the areas of retina in which they arise but also physically. Tension is the force which occasion bitemporal hemianopia and pressure that which produces nasal field defects.

With the development of neurological surgery during the last half century there has been much careful work on the visual field defects produced by lesions in the sellar neighbourhood. The combined studies of ophthalmologists, neurologists, and neurosurgeons have added to knowledge and refined techniques have led to extremely accurate charting of abnormalities in the fields. However, as will be described, certain features of the field disturbances associated with tumours related to the optic chiasma remained unexplained. It is believed that a reexamination of the anatomy of the optic chiasma may throw some light on these problems and the results of such an examination and its implications form the basis of this paper.

The term hemianopia indicates a defect in half of the visual field in one eye, usually a vertical half, and it may be incomplete. Hemianopia may be homonymous right or left when corresponding half fields in each eye are affected. This results from a lesion of the optic tract, geniculocalcarine pathway or striate cortex. Such a defect is a single one of the binocular field. On the other hand, hemianopia may be heteronymous when both outer or both inner half fields are involved. Such defects will usually be secondary to a lesion of the optic chiasma and will be bitemporal if the crossing fibres are involved, or binasal if the uncrossed are affected. Here the defects involve both sides of the binocular field. It is believed that the mechanisms responsible for binasal and bitemporal hemianopia are distinct and related to chiasmal anatomy and they will be discussed after the anatomy of this area has been reviewed.

\section{METHODS}

In 25 postmortem subjects following removal of the calvarium the frontal dura mater was incised transversely, the falx cerebri sectioned, and the frontal lobes elevated to display the optic nerves, optic chiasma, and anterior parts of the optic tracts as well as related arteries. The arachnoid mater was separated from the chiasmal region and, with a nerve hook the resistance to deformation and displacement of the nervous structures was estimated. The tension in the medial and lateral portions of the optic nerves was determined by noting the gap which developed 
in these after appropriate section. The effect on these gaps of subsequent chiasmal and tract section was also determined. Photographs were made to demonstrate the results.

In 20 cases after its removal from the skull and fixation in $5 \%$ formalin solution the chiasma was macerated in $30 \%$ alcohol for periods of from one to three weeks. The specimens were then dissected under the microscope (magnification $\times 10$ ) and the appearances sketched and photographed. The microscopic anatomy of the optic nerves, chiasma, and tracts was reviewed in sections, stained with haematoxylin and eosin, haematoxylin and Van Gieson, and Loyez's stains.

\section{ANATOMY OF OPTIC CHIASMA}

Not only embryologically but topographically the optic chiasma is an integral part of the brain (diencephalon). While it projects inferiorly and anteriorly into the cisterna basalis, it forms part of the third ventricular wall between the lamina terminalis and the tuber cinereum (Fig. 1). Moreover, its posterolateral angles are continued into the optic tracts, which are themselves attached to the cerebrum and cerebral peduncles, and these structures are clothed in a well-marked pial layer which passes anteriorly on to the optic nerves where these join its anterolateral angles. The dimensions of this junctional zone between the optic nerves and optic tracts vary. Whitnall (1932) gives as mean values $13.28 \mathrm{~mm}$ transversely, $8 \mathrm{~mm}$ anteroposteriorly, and 3 to $5 \mathrm{~mm}$ in thickness. Its anteroposterior relationship to the sella turcica varies. According to Schaeffer (1924) in $79 \%$ the chiasma lies almost wholly over the diaphragma sellae overlapping the dorsum to a slight extent, and in $12 \%$ it lies wholly over the diaphragma sellae; in $5 \%$ it lies in part in the sulcus chiasmaticus of the skull base and in part on the diaphragma sellae, and in $4 \%$ it lies on and behind the dorsum sellae. Clearly the more posterior the position of the chiasma, the longer will be the intracranial portions of the optic nerves-their length varying from 6 to $21 \mathrm{~mm}$ (Zander, 1896). Of the other relations of the chiasma, that to the neighbouring arteriesinternal carotid, anterior cerebral, and anterior communicating - is of particular importance. As pointed out by Bull (1956), the vertical height of

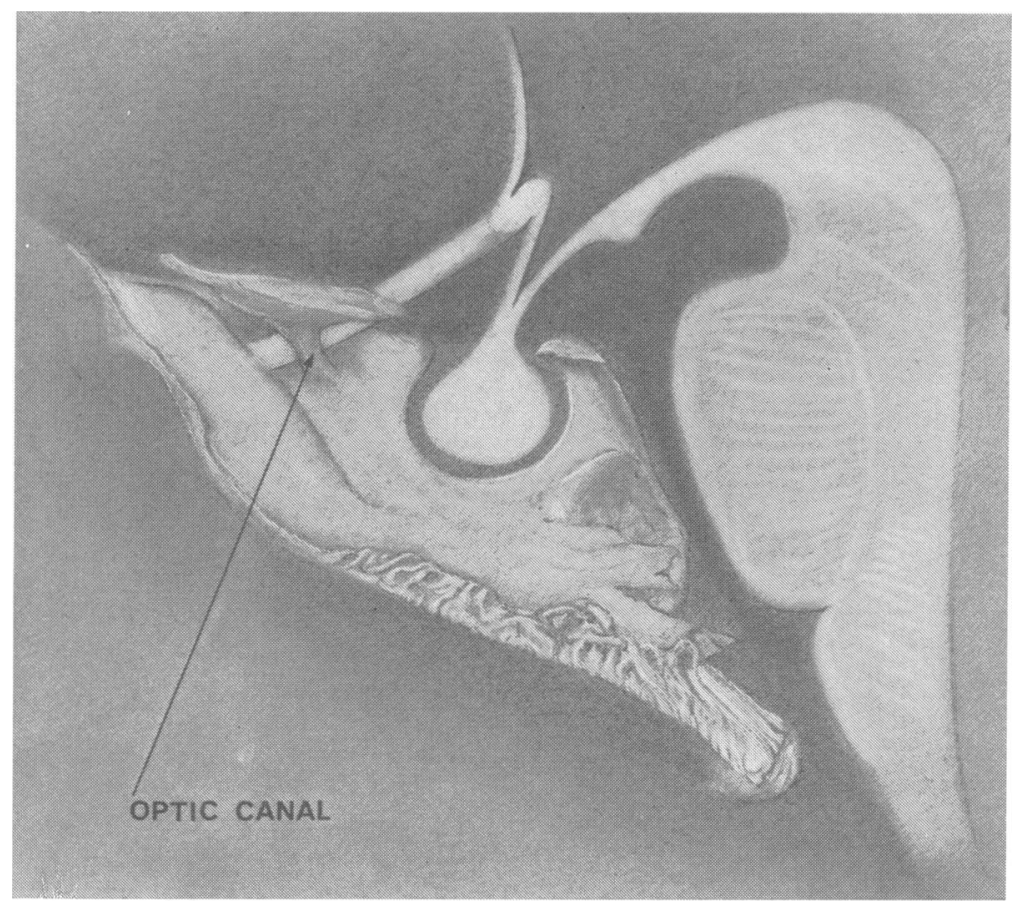

FIG. 1. The attachments of the optic nerve at optic canal and chiasma. 

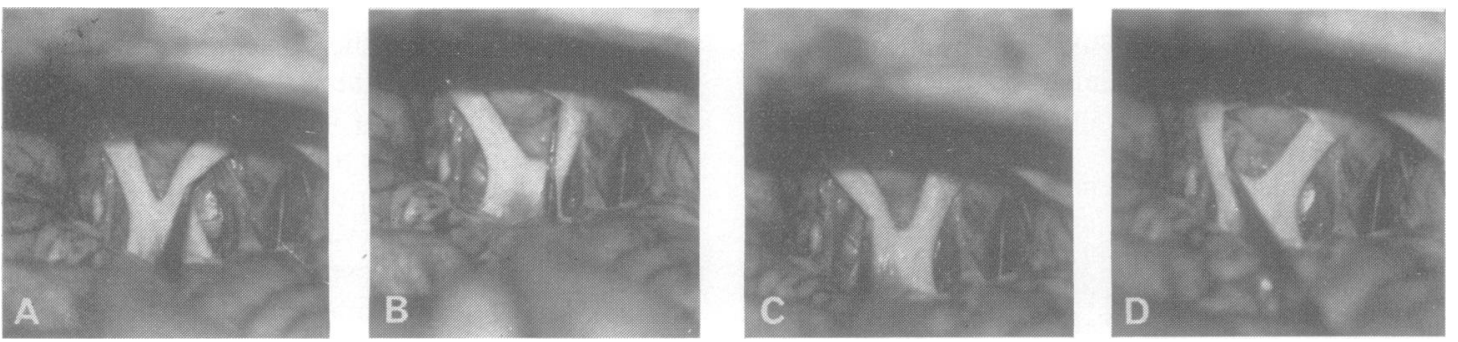

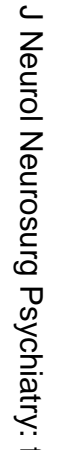

FIG. 2. Photograph of dissection to show differences in resistance to deformation and displacement in lateral and medial fibres of optic nerves.
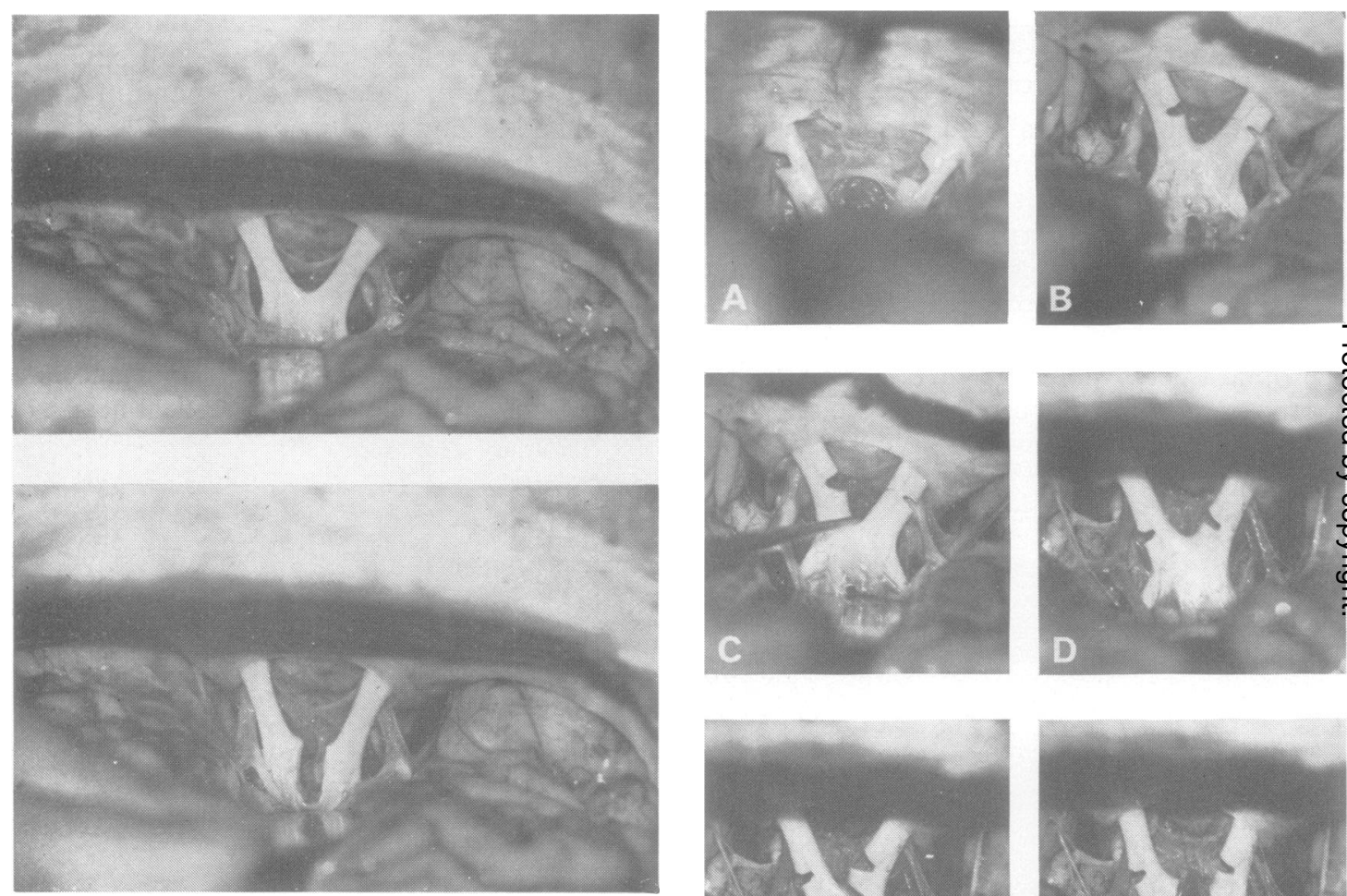

FIG. 3. Photograph showing effects of sagittal section of chiasma.
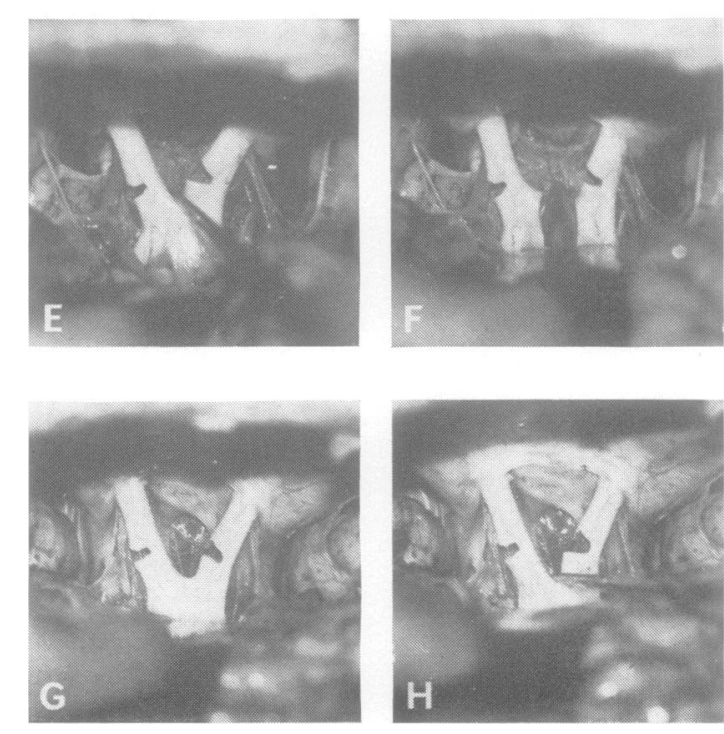

FIG. 4. A. Anterior nerve sections showing wider gaping of medial incision. B and C. Intermediate sections showing wider gaping of medial incision and its increase with chiasmal retraction. $\mathrm{D}, \mathrm{E}$, and $\mathrm{F}$. Posterior section with wider gaping of medial incision, after chiasmal retraction, the situation being reversed after chiasmal section. $\mathrm{G}$ and $\mathrm{H}$. Posterior section with wider gaping of medial incision increased by chiasmal retraction.

$\overrightarrow{\vec{B}}$ 
the chiasma above the skull base varies, just as does its distance posterior to the tuberculum sellae, according to the length of the optic nerves. It is difficult to estimate the former distance, but the latter he states to be 10 to $23 \mathrm{~mm}$.

DIFFERENCES IN RESISTANCE TO DEFORMATION AND DISPLACEMENT IN OPTIC NERVES AND TRACTS After exposure of the area in the manner described, pressure against the lateral margin of the optic nerve and chiasma reveals, at first, a low resistance to deformation and to the medial displacement of the nerves, the chiasma becoming relaxed (Fig. 2, A and C). Pressure against the medial margin of the nerve reveals a higher resistance to deformation and lateral displacement, the chiasma being stretched and the opposite optic nerve drawn medially (Fig. 2, B and D). These differences are particularly marked immediately anterior to the chiasma and increased with posterior retraction of that structure. Posterior displacement of the chiasma is itself strongly resisted. There is relatively little resistance to displacement of the optic tracts which retract like brain tissue itself. There is little resistance to downward displacement of either medial or lateral fibres of the optic nerves, the degree of such displacement being, however, restricted by the underlying cranial floor. However, when upward displacement is attempted, this is resisted more strongly by the medial than by the lateral fibres.

DIFFERENCES IN TENSION IN OPTIC NERVE FIBRES AS INDICATED BY RESULTS OF SECTION AT VARIOUS SITES (1) Sagittal section of optic chiasma (Fig. 3) After sagittal section of the chiasma separation of the cut surfaces occurs and the optic nerves move laterally, the nerves and tracts moving closer towards anteroposterior alignment. The gap between the cut surfaces measured 4 to $5 \mathrm{~mm}$, yet the separation of the lateral chiasmal margins amounted to only 2 to $3 \mathrm{~mm}$. This implies that chiasmal fibres are in part retracted into the optic nerve and tract by tension within these.

2. Partial section of optic nerves (Fig. 4, A, B, C, G, H; Fig. 5, A, B, C, D) The medial half of one optic nerve and the lateral half of the other were sectioned. This was carried out at anterior, intermediate, and posterior levels in the nerves of different specimens. In almost all cases, and at all levels, but more markedly at posterior ones, the incision on the medial side of the nerve gaped more widely than that on the lateral side. The most posterior incisions were twice as wide medially as laterally, and it was noted that they tended to open up in a rectangular manner on the medial side instead of remaining wedgeshaped as in the case of lateral incisions. Gentle posterior retraction on the chiasma made the difference considerably more obvious. The gap as measured at the medial margin of the nerve varied from 1.5 to $4 \mathrm{~mm}$, and at the lateral margin of the nerve 0.5 to $2.5 \mathrm{~mm}$. After posterior retraction of the chiasma, the medial measurement was 3 to $5 \mathrm{~mm}$, and the lateral one 1 to
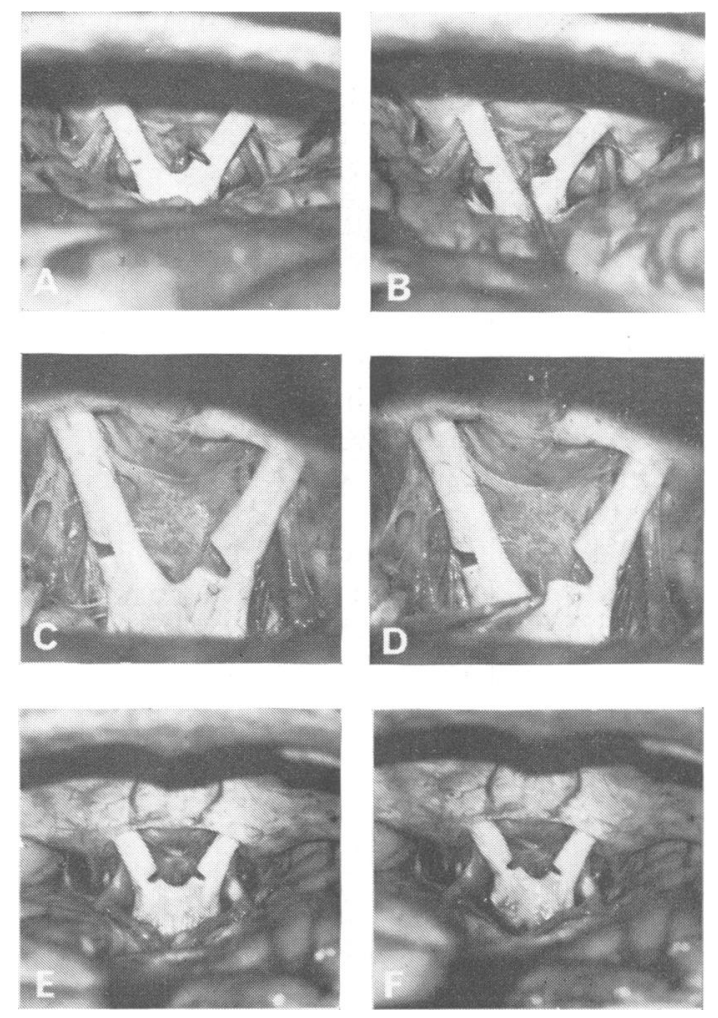

FIG. 5. A, B, C, and D. Posterior section of medial and lateral fibres and effects of chiasmal retraction upon them. E and F. Bilateral section of medial fibres showing decrease in gap in right nerve end and increase in that in the left nerve after left tract section. 
$3 \mathrm{~mm}$. These findings indicate that when the chiasma is exposed in the manner described, tension develops in the optic nerves, and is more marked in their medial than in their lateral fibres. The degree of tension will vary from case to case, but is invariably increased by chiasmal retraction.

\section{Partial section of optic nerves combined with} chiasmal and tract sections (Fig. 4, D, E, and F; Fig. 5, E and F) If sagittal section of the chiasma is carried out on a specimen in which the medial half of one optic nerve and the lateral half of the other has been sectioned, it is found that the gap on the lateral side widens, and that on the medial side diminishes. In such a case, much of the tension is transferred to the lateral fibres, and the usual state of affairs is reversed. If such chiasmal section is carried out on a specimen in which the medial halves of both optic nerves have been sectioned, the gap on both sides diminishes in extent. Again if after section of the medial halves of both optic nerves, the optic tract is divided on one side, the gap in the opposite optic nerve diminishes in width, tension having been reduced in its crossing fibres.

It is believed that this series of observations, like the preceding ones, indicates that when the frontal lobes are elevated or the chiasma retracted posteriorly, an increase in tension develops in the optic nerve fibres, and that this tension is considerably greater in the medial crossing fibres than in the lateral uncrossed ones. It should be pointed out that, while some of the measurements described were made on the exposed specimens, the majority were made for greater accuracy from photographs. The magnification of these varied to some extent and the actual measurements are not therefore reliable; however, the plates do reveal the differences in the gaps which develop in the medial and lateral groups of fibres in the nerves after fractional section and the effects of chiasmal and tract sections upon these.

INTERNAL ANATOMY OF OPTIC CHIASMA Information on this subject is less detailed than might be expected. It is now believed that the ratio of crossed to uncrossed fibres in the chiasma is $53: 47$ and that the number of fibres of diameter greater than $0.5 \mu$ in each optic nerve is in the order of 1.1 million (Kupfer et al., 1967). Moreover, it is accepted that the nasal fibres in the optic nerves cross anteriorly in the chiasma and the macular ones posteriorly. Further, it is stated that the more anteriorly placed nasal fibres loop forwards into the termination of the contralateral optic nerve before turning posteriorly, while the more posteriorly placed and lateral of those fibres are described as looping backwards into the origin of the ipsilateral optic tract before crossing in the chiasma to the contralateral one. Anatomical illustrations to support these statements are lacking in a wide selection of anatomical, neurological, and ophthalmological works. Reference is, however, frequently made to the writings of Henschen (1890 and 1892) and Wilbrand and Sänger (1904). In these beautifully illustrated works, information concerning the preparation of the specimens is scanty, though Wilbrand and Sänger (1904) describe their plates as 'being true to nature'. The plates appear to be drawings rather than photograph and designed to show, in one figure, the finding from the examination of a number of horizonta sections of the chiasma. Polyak (1957) provides \& more complete account of chiasmal anatom with a description of the methods by which hig specimens were prepared, though again the illustrations appear to be drawings rather tha photographs. While it is clear that the finer details of fibre arrangement in the chiasma can only be determined by histological methods, it seemed possible that dissection of this structure under magnification could provide a better threedimensional view of the general internal arrangement of the fibre bundles and the resulting physical properties of this decussation-unique because of its anatomical site outside, though a part of, the central nervous system.

Chiasmal dissections The pial sheath of the chiasma was noted to be extremely well developed particularly on its superior aspect. As had been noted during dissections of the optic nerve in connection with an investigation of intraneural plexus formation (O'Connell, 1936) the fibres of the optic nerve could be separated into parallel rounded bundles without intercommunication. The same was true of the anterior part of the optic tracts, the larger bundles here being flattened with concave medial and convex lateral 


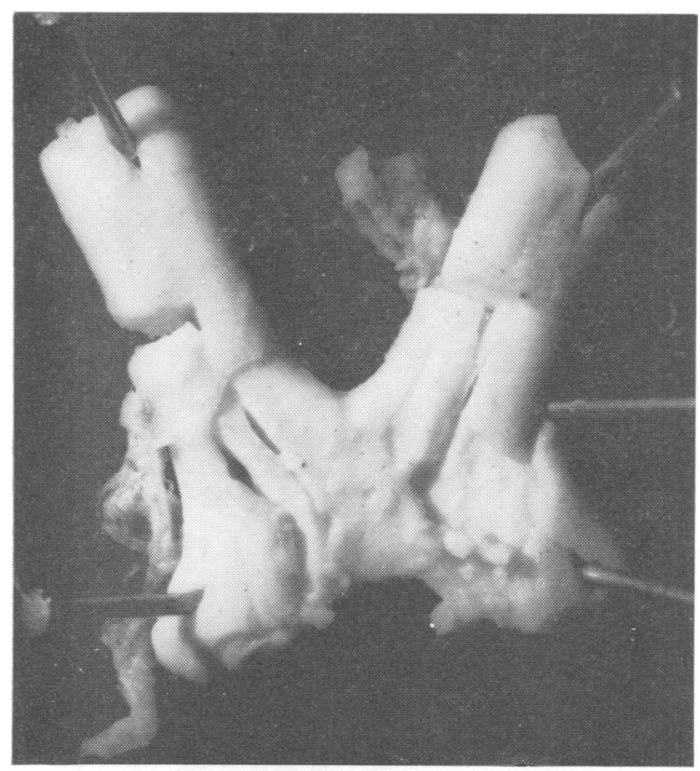

(a)

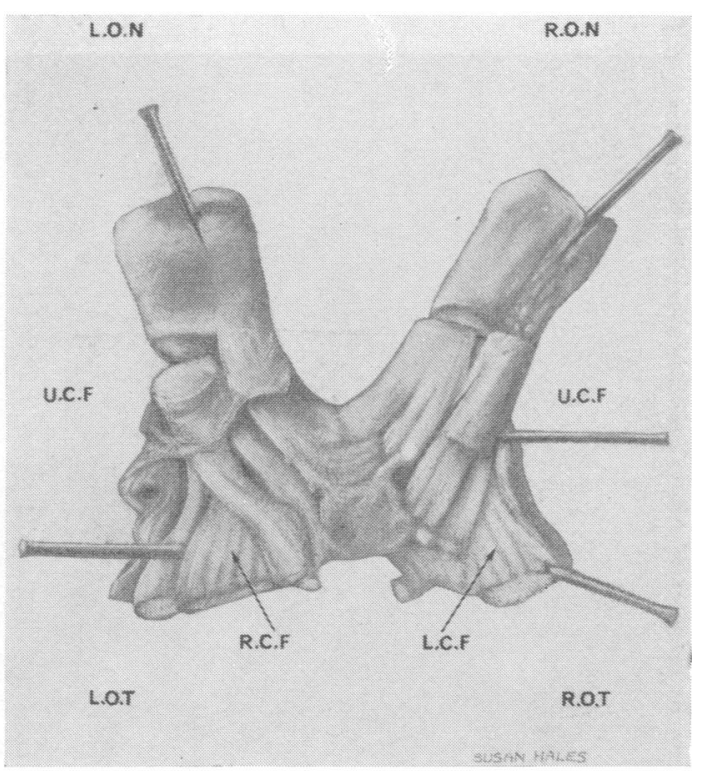

(b)

FIG. 6. (a) Photograph of superficial dissection of superior aspect of chiasma showing crossed and uncrossed fibres. At the origin of the optic tract the latter separate into medial and lateral bundles with the crossed fibres from the opposite nerve between them. (b) Drawing of above dissection. L.O.N. and R.O.N.-optic nerves. L.O.T. and R.O.T.-optic tracts. U.C.F.- uncrossed fibres. L.C.F. and R.C.F.-crossed fibres with side of origin.

surfaces. The lateral fibres in the nerve could be traced through the lateral chiasma into the lateral tract. Here a division occurred so that the superior fibres passed to the superomedial aspect of the emerging crossed fibres from the opposite side (Fig. 6).

Dissection of the superior aspect of the chiasma revealed that the superior crossing fibres on each side spread out in a triangular sheet of bundles with its apex at the termination of the optic nerve and its base at the midline (Figs 6 and 7). Anteriorly were the relatively short medial fibres crossing in the anterior portion of the chiasma and posteriorly and laterally the lateral crossing fibres passing to the posterior margin of the structure (Fig. 8). Fibre bundles of 1.5 to $2 \mathrm{~mm}$ in diameter could be readily traced medially under the dissecting microscope. However, as the central area of the chiasma was approached, these split into smaller bundles until diameters of $0.2 \mathrm{~mm}$ or less were reached, and further dissection became impossible as it led to their being torn. Beneath the most super- ficial layer of crossing fibres, further similar layers, four in number, can be made out.

Dissection of the inferior surface of the chiasma displays the crossed fibres emerging from it in the midline throughout its anteroposterior extent and passing into the optic tract on each side (Fig. 9). Some 15 bundles of a diameter greater than $0.25 \mathrm{~mm}$ could be made out and, as they are traced into the tract, some eight flattened bundles are formed. As in the case of the crossed fibres entering the chiasma from the optic nerve, so in the case of those leaving it to enter the tract, superimposed layers of bundles could be separated (Fig. 10).

In carrying out the dissections of the inferior surface of the chiasma attempts were made to separate the non-visual fibres of the supraoptic decussations-including Gudden's commisure. Separation of a considerable bundle of fibres which, under the dissecting microscope, appeared quite distinct from the visual ones was possible. However, this method of examination is a 

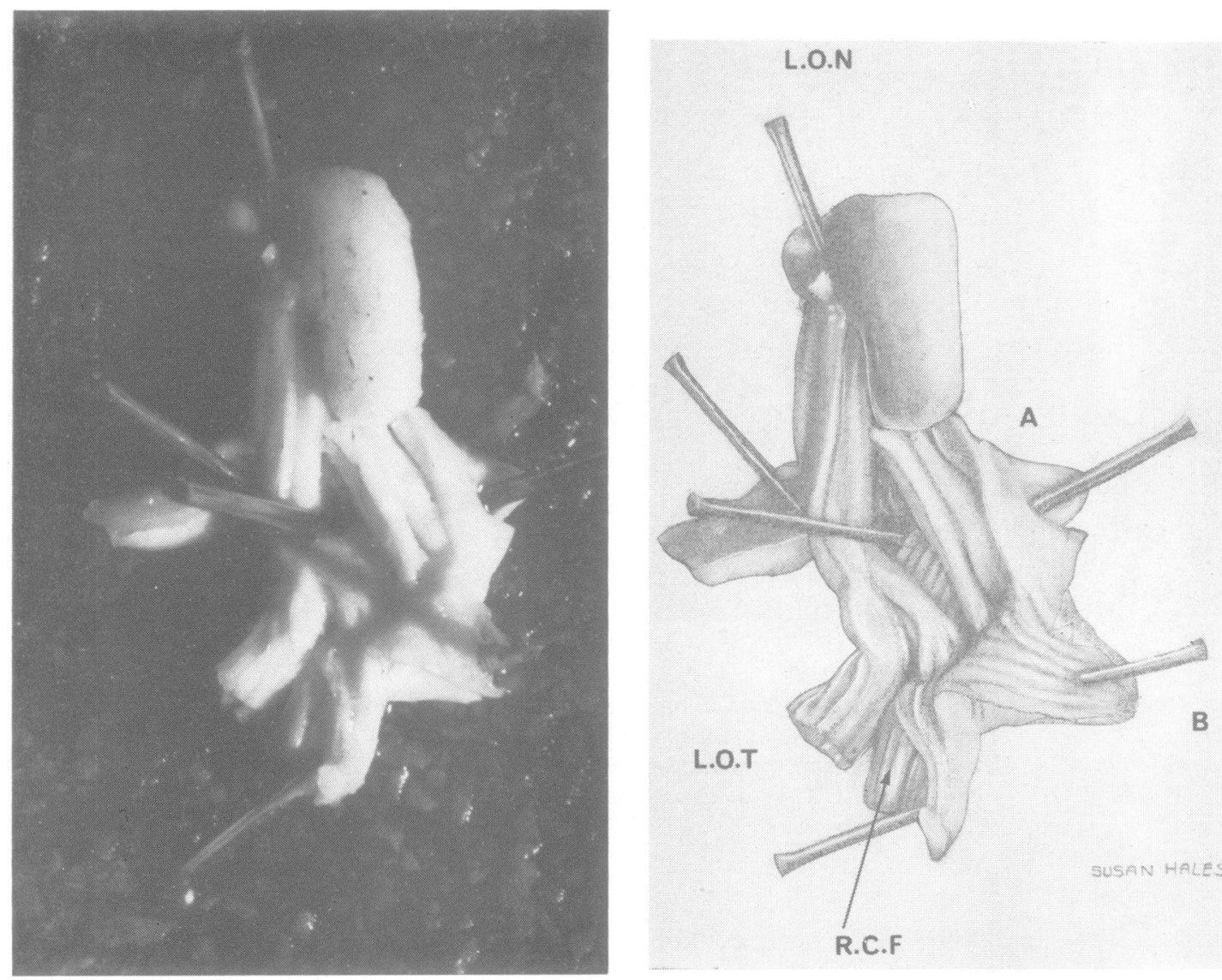

(a)

(b)

FIG. 7. (a) Photograph of dissection of superior surface of left half of chiasma. A deeper layer of crossing fibres has been exposed by section of a superficial group at the termination of the nerve and retracting it medially and inferiorly. (b) Drawing of above dissection. L.O.N. and L.O.T.-left optic nerve and tract. A. - point of section of superficial group of crossing fibres. B.-distal retracted end of this group. R.C.F.-crossed fibres from right nerve.

relatively crude one and permits statements concerning gross anatomy only.

MICROSCOPIC ANATOMY OF OPTIC CHIASMA A limited examination alone was carried out. In the longitudinal sections of the chiasma and attached nerves and tracts, longitudinally running bundles could be made out in both nerve and tract and the presence of fibrous tissue among the bundles of the nerve and its absence among those of the chiasma and tract was con- firmed. Again transversely directed fibres were seen in the chiasma, the arrangement in bundles being no longer obvious. In the median sagittal plane the decussation of fibres could be clearly seen (Fig. 11). In the lateral chiasma between the termination of the optic nerve and the origin of the optic tract it became impossible to trace fibres in continuity-doubtless because those seen were entering or leaving the plane of the section. Here some fibres were cut transversely $N$ and others longitudinally for short lengths and N curved groups of fibres could be seen. No 


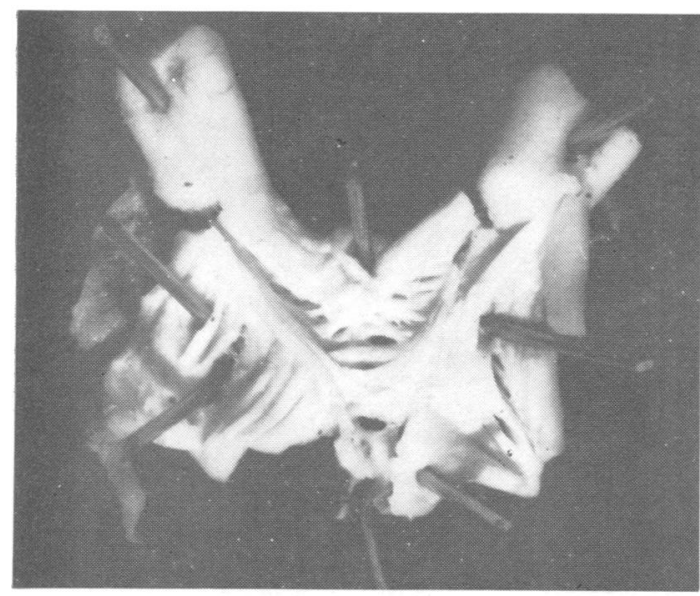

(a)

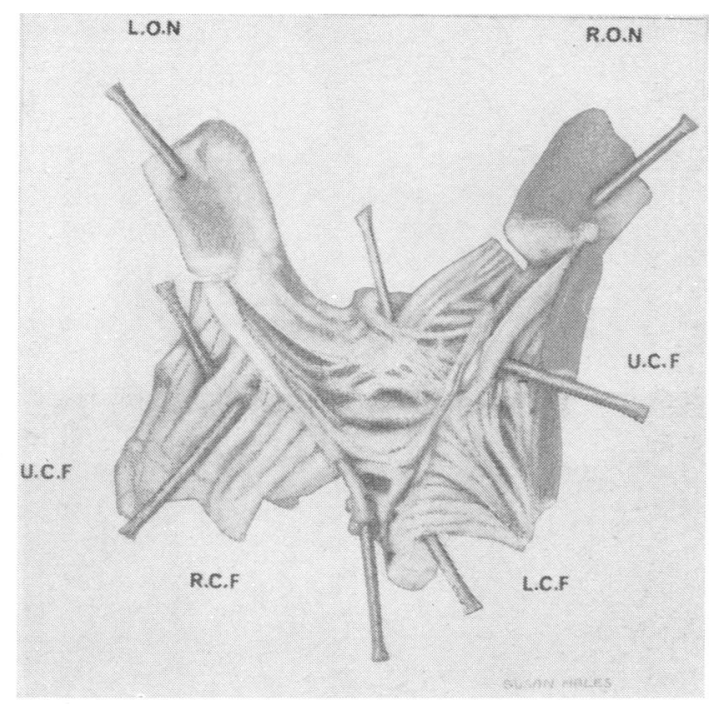

(b)

FIG. 8. (a) Photograph of deeper dissection of superior surface of the chiasma showing interweaving of bundles of crossing fibres, those most laterally placed in the nerve crossing posteriorly. (b). Drawing of (a). L.O.N. and R.O.N.-optic nerves. U.C.F.- uncrossed fibres. R.C.F. and L.C.F.—crossed fibres with side of origin.

attempt to carry out a detailed microscopic study was made.

\section{DISCUSSION}

A structure such as a nerve coursing between two points of attachment will be capable of maximum displacement transversely midway between these points and minimal displacement at the attachments. Should a mass arise along the course of the nerve, it is perhaps better to regard the latter as being stretched as a result of displacement rather than as being compressedunless, of course, it is trapped between the mass and some related structure.

Anteriorly, the intracanalicular portion of the optic nerve in its 4 to $9 \mathrm{~mm}$ course is firmly attached around its circumference in the optic canal by adhesion between its sheaths of arachnoid and dura mater (Fig. 1), the nerve and its sheaths filling the canal. The attachment lies further anteriorly than it at first appears because of the dural fold which passes from the anterior clinoid process to the tuberculum sellae, which may be $5 \mathrm{~mm}$ or more in width (Fig. 12). The internal carotid artery lies beneath the nerve at this point and medial and, to a lesser extent, lateral mobility of the nerve at the level where a sellar mass would impinge upon it is greater than is the case anteriorly at the optic canal.

Posteriorly, the nerve is attached to the anterolateral angle of the chiasma and here the uncrossed fibres take an uninterrupted course through it into the optic tract. The crossed fibres, however, spread out and intimately interweave in the midline of the chiasma with those of the opposite optic nerve before being gathered together to enter the contralateral optic tract. This interweaving produces a degree of resistance to displacement in the medial fibres of the optic nerve and in the chiasma which, as has been shown, is greater than in their lateral fibres. Moreover, the tension which develops in the medial fibres of nerves and chiasma anchors the latter and any displacement of the chiasma applies a stretching force to the bundles of decussating fibres within it. Thus the medial and lateral fibres of the optic nerves and chiasma differ not only in the areas of retina from which they arise but also in their physical properties 
(a)

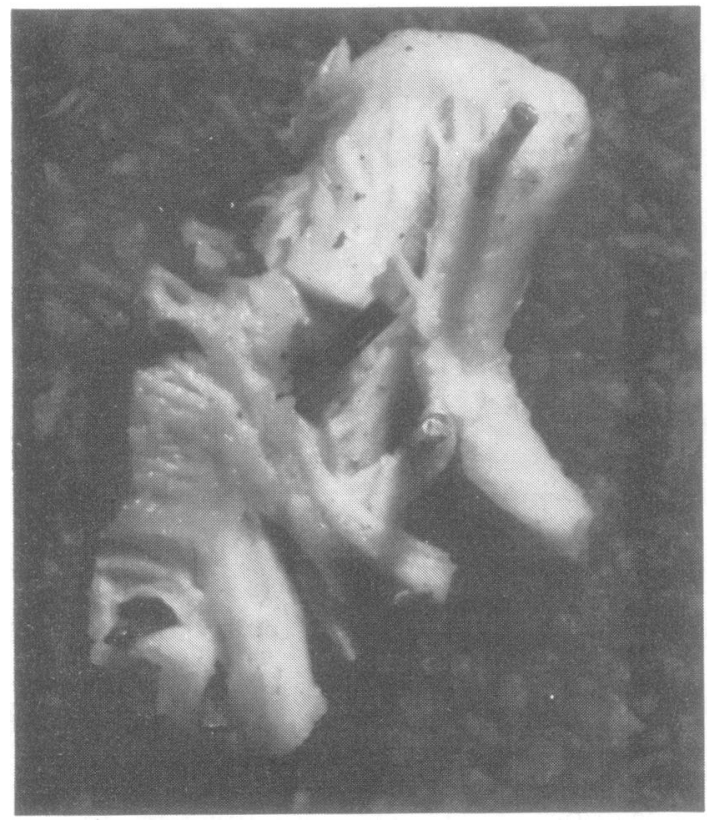

L.O.N

(b)

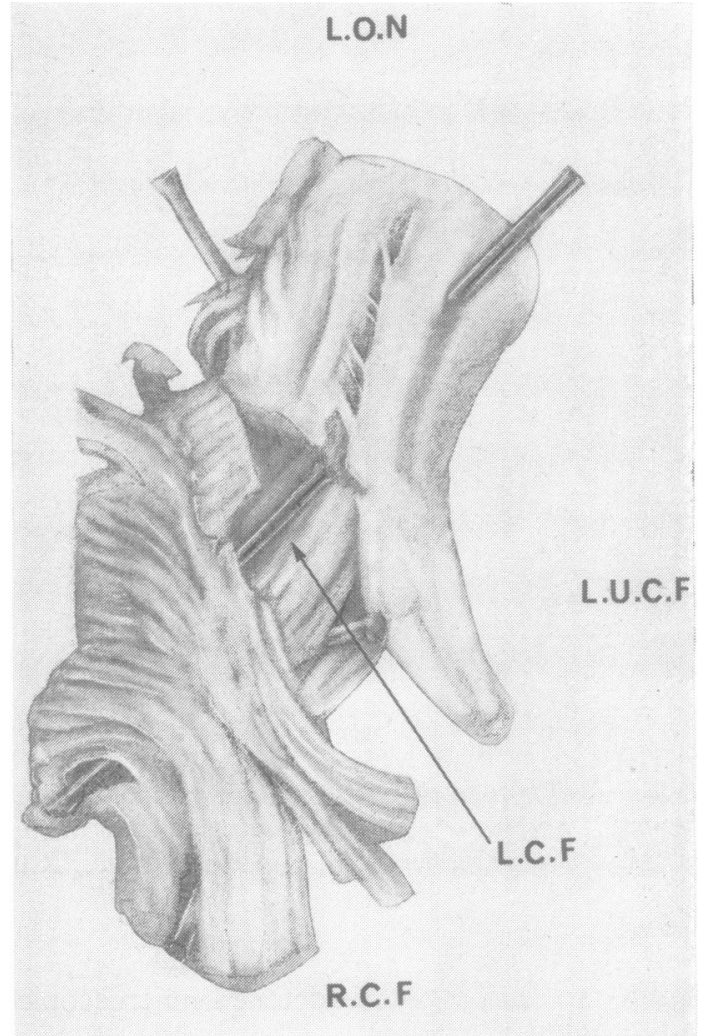

FIG. 9. (a) Photograph of dissection of inferior surface of left half of chiasma showing uncrossed fibres laterally and crossing fibres medially, the crossed fibres from the right side emerging in the mid-line and passing into the left tract. (b) Drawing of (a). L.O.N.-left optic nerve. L.U.C.F.-uncrossed fibres. L.C.F.-left crossing fibres. R.C.F.-right crossed fibres.

because of their differing posterior attachments. The possible relationship of these observations to heteronymous hemianopia will now be considered.

BINASAL HEMIANOPIA Binasal hemianopia has been discussed in a recent paper (O'Connell and du Boulay, 1973). The literature was reviewed and the commonly held view that the development of a binasal hemianopia necessitated bilateral and symmetrical lesions on the lateral aspects of the optic nerves described. It is noteworthy that, before the development of neuro- logical surgery, numerous descriptions of the postmortem finding of grooving of the optic nerves, tracts, and chiasma by the Willisian vessels in the presence of tumours in the sellar region had appeared; however, these changes had not for the most part been related to visual field abnormalities. The rarity of the surgical confirmation of such findings is thought to be due to the relatively limited operative as distinct from necropsy exposures. However, utilization of modern methods of investigation can demonstrate the detailed relationships of a tumour to the Willisian vessels and basal visual pathways. 


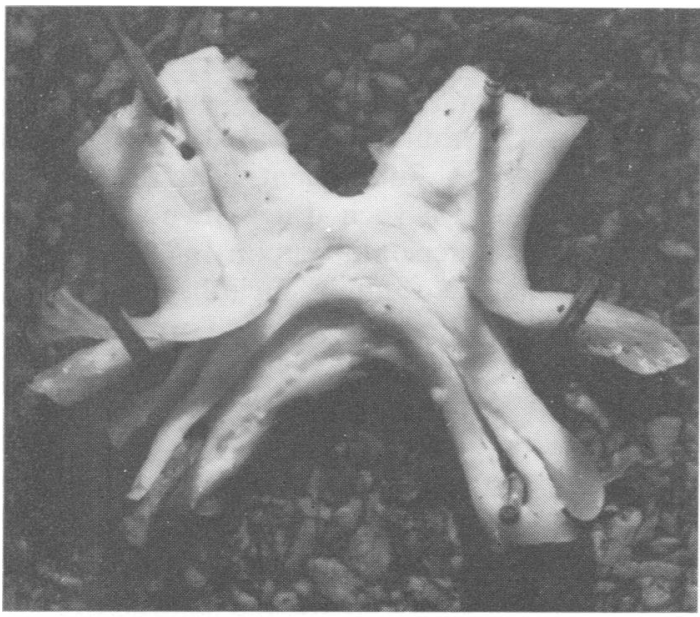

(a)

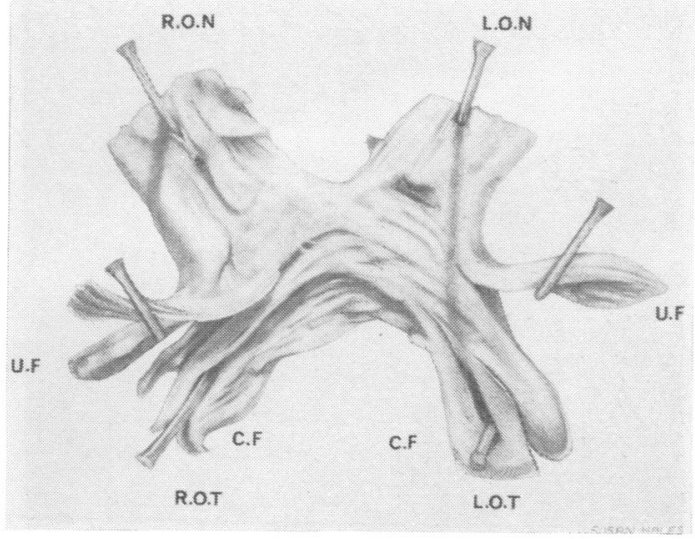

(b)

FIG. 10. (a) Photograph of dissection of inferior surface of chiasma showing uncrossed fibres laterally and crossed fibres from each side emerging in midline and passing into optic tracts. (b) Drawing of (a). R.O.N. and L.O.N.—optic nerves. R.O.T. and L.O.T.—optic tracts. U.F.-uncrossed fibres. C.F.—crossed fibres.

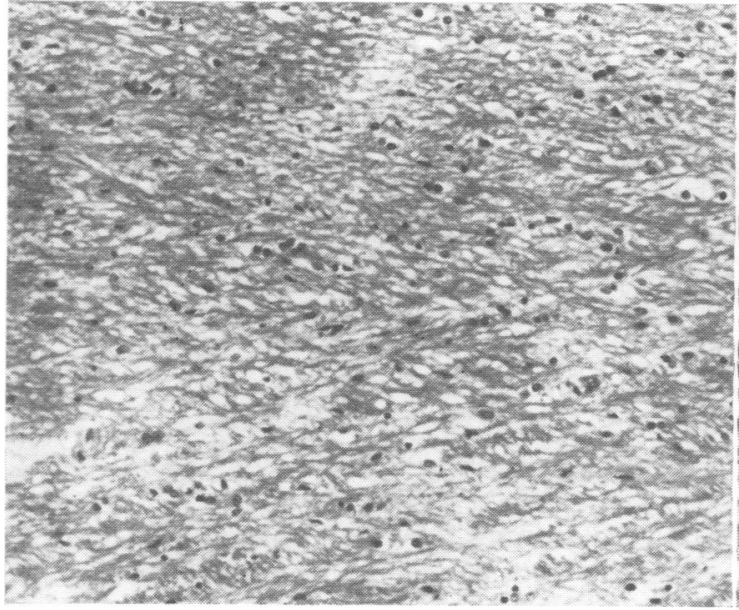

(a)

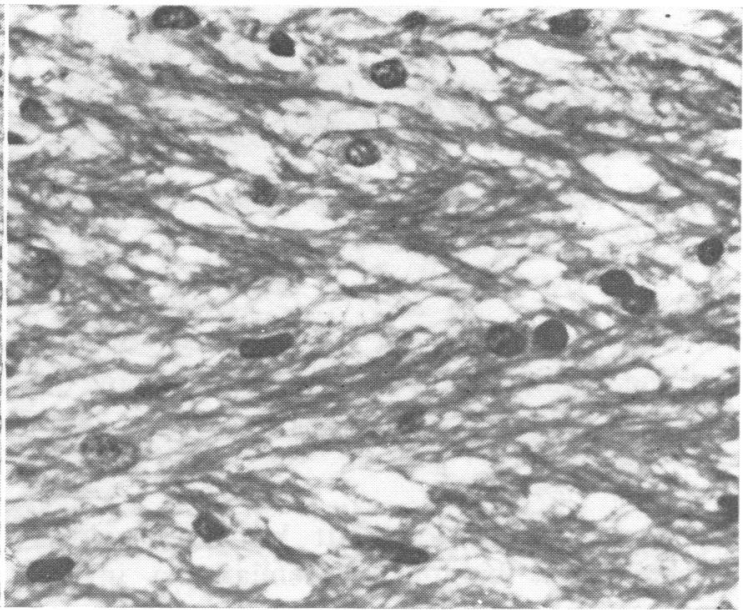

(b)

FIG. 11. Transverse section through posterior portion of optic chiasma: $H$ and $E:(a) \times 100$; (b) $\times 400$.

In three cases described these relationships were demonstrated and nasal field defects explained. It is thought that as a result a displacement of the internal carotid or anterior cerebral artery against the optic nerve or chiasma, or, the reverse, a displacement of the nervous structures against the vascular ones, or a combination of the two, compression of the nervous structures occurs and grooving of the lateral uncrossed fibres produces the field defect. The superolateral fibres of nerve and chiasma are most intimately related to the vessels mentioned. These will pro- 


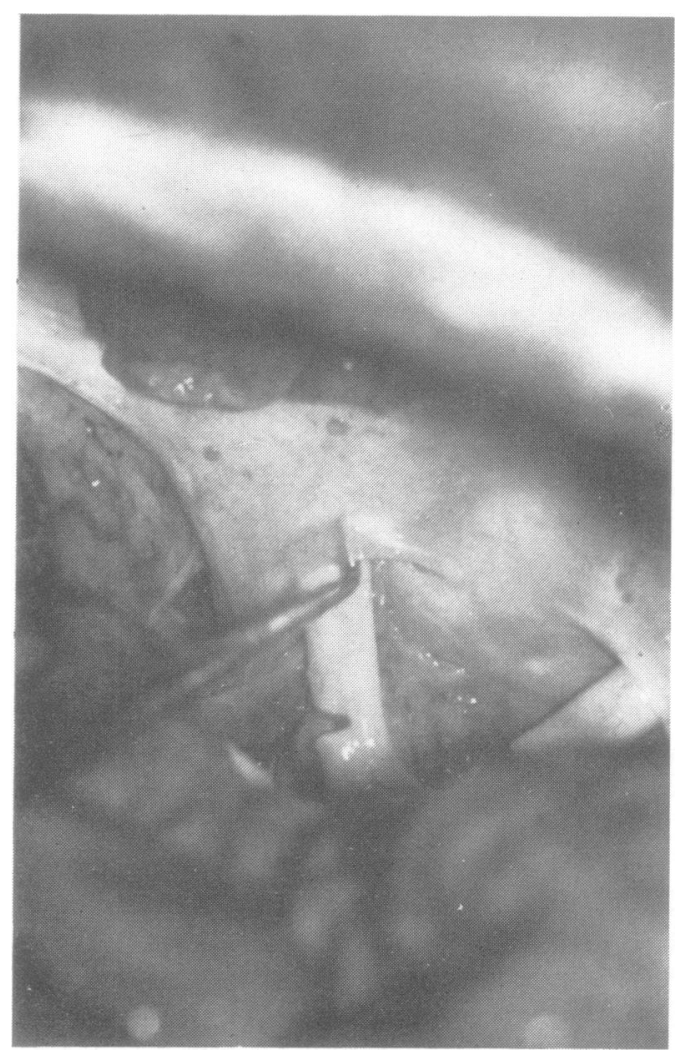

FIG. 12. Photograph of dural fold covering the optic nerve after its escape from optic canal.

tect the medial fibres and be cut into by the stretched pulsating vessels as though by a blunt saw.

BITEMPORAL HEMIANOPIA It has been widely accepted that bitemporal hemianopia in the presence of a tumour related to the sella turcica is due to compression of the crossing fibres in the optic chiasma. However a number of authors have questioned this view. Fisher (1913) regarded it as glib and asked 'How can a tumour originating in the interpeduncular fossa produce the definite and precise effects of a sagittal section of the chiasma? It would take almost a knife edge to do this'. He believed that the visual phenomena in many cases were explained by traction effects on the visual pathways as the tumour extended upwards behind the chiasma and between the optic tracts. A tumour here would, he thought, stretch the decussating fibres situated in the midline and occasion a bitemporal hemianopia without in any way dragging on the uncrossed fibres. Subsequent field disturbance would result from such dragging on the optic nerve or tract, depending upon the position of the major mass of tumour. Fleischer (1914) supported the traction hypothesis. Traquair (1917) discussed the matter in detail and also questioned how the rounded dome of a tumour could produce a limited anteroposterior knifeedged pressure such as would be required to produce the clear-cut field defects often found. He decided that the evidence showed that such precise pressure is not required to produce these field defects. He was particularly perplexed by the cases of bitemporal hemianopia not due to space occupying lesions which he had studied and stressed that these must be considered in any attempt to explain the mechanism of this type ofo field defect. He thought that the traction explana tion was open to the same objections as the pres sure one. The third possibility considered was local intoxication arising from a chiasmaP neuritis, local inflammatory conditions, or the products of tumour degeneration or disturbance of endocrine secretion. He admitted, however that in tumour cases mechanical factors could be important. Walker and Cushing (1918) in discussing bitemporal hemianopia pointed out that the absence of an upper nasal defect when an upper temporal one has developed means that pressure is not the sole factor at work; tension and strangulation in the crossing fibres are additional factors. Tension augments pressure and both lead to strangulation, the latter probably occasioning the physiological block. They suggested that the delay in development of a nasal defect is occasioned by the additional pressure and distortion necessary before the uncrossed fibres are affected in the same way. However, in the last half century it appears that chiasmal compression has become accepted as the cause of bitemporal hemianopia in tumour cases. Forty years after his earlier communication Traquair in the seventh edition of his Clinical Perimetry (Scott, 1957) appears to have accepted pressure as the most attractive hypothesis. He did this by taking the view that pressure acted not on nerve fibres directly but on 
their vascular supply, thus occasioning ischaemia.

Yet the view that compression is the factor responsible for bitemporal hemianopia is no more readily acceptable now than it was half a century ago and a number of problems arise in connection with the hypothesis. Although a part of the central nervous system, the optic nerves and chiasma lie outside it, and might be expected to respond to compression like other cranial nerves. However, the hypersensitivity of its crossing fibres and the relative insensitivity of the uncrossed ones are in contrast with the insensitivity to pressure of all the fibres of, say, the facial nerve in the presence of large acoustic neurinomata. This may be so stretched and compressed as to be unrecognizable and yet no facial paresis may be present. Moreover, when in the latter cases a facial paresis does appear, it affects all parts of the face equally. Regarding the nerves and chiasma as part of the central nervous system, the reaction of their fibres to compression is still in contrast with those of the brain and spinal cord. The clinical localization of mass lesions in the cranium and spinal canal depends upon the functional disturbance occasioned by compression of the areas most closely related to the mass. There is here no evidence of differing sensitivities of neurones and the clinical picture depends upon the intimacy of the relationship between eloquent areas and the compressing agent. The dimensions of the chiasma are small and yet repeatedly the rounded dome of a sellar tumour, although varying in its exact position, will produce a bitemporal hemianopia, with or without temporal scotomata and only secondarily and at a late stage will a nasal field loss develop. It might be expected that such a mass between the optic nerves compressing their inferior and medial fibres would give rise to a defect in the superolateral visual fields which would progress inferiorly and medially like a total solar eclipse until the field loss was complete. However, in spite of the smallness of the target and the inevitable variation of the site of the application of the compressing force, the result is repeatedly the same, commencing with the picture of a progressive sagittal section of the chiasma in the midline. After a bitemporal hemianopia is established there is frequently a considerable time interval before vision begins to fail in the inferior nasal quadrants and a fur- ther delay before the upper nasal quadrants become involved. Moreover as has been said, binasal hemianopia is by itself extremely uncommon.

THE TENSION HYPOTHESIS As has been seen the intracranial portion of the optic nerve has two attachments. Anteriorly, the whole circumference of the nerve is attached in the optic canal by adhesion between its arachnoid and dural sheaths and the bone. Posteriorly, the lateral fibres of the nerve are continued into the optic tract and the attachment is a relatively lax one. The medial fibres crossing in the chiasma spread out through it and interweave with the medial fibres of the opposite nerve before being gathered together into the contralateral optic tract. This firm posterior attachment of the medial fibres lies in the median sagittal plane of the chiasma where the interweaving of nerve bundles is at microscopic (nerve fibre) level. Over half a million nerve fibres from each optic nerve cross one another in small groups, if not as individual fibres, at angles of up to $90^{\circ}$ in the chiasma. Tension developed in these crossing nerve fibres as a result of displacement of optic nerves or chiasma would lead to mutual compression and possibly even section. There is a further structural reason for chiasmal sensitivity to tension. It has been shown that the tensile strength of peripheral nerves is particularly dependent on the endoneurium - not the epi- or perineurium, nor the axons nor their myelin sheaths (Sunderland and Bradley, 1961a). Spinal nerve roots, in which there is a scarcity of endoneurial collagen (Gamble, 1964), resist longitudinal tension less well than peripheral nerves because of this structural difference. In the case of the optic chiasma the protection from tensile forces is even less good. While in the optic nerve there are fibrous tissue septa between the groups of funiculi, in the chiasma and tract collagen is absent and, as in the rest of the central nervous system, the supporting tissue is purely glialespecially astrocytic. Thus the posterior attachment of the medial fibres of the optic nerve is not only a firm physical one; because of the angled crossing of large numbers of closely packed fibres in the median plane of the optic chiasma and the lack of collagenous support for them, it is to be 
expected that the fibres in the area will be highly sensitive to injury by tension.

Irrespective of the exact site of impingement of a tumour on the nerves or chiasma, displacement of them applies tension to the crossing fibres in the midline and defects of function result. Displacement laterally of the optic nerves and displacement posteriorly or elevation of the chiasma leads to an increase in tension in the crossing fibres in both nerve and chiasma (Fig. 1). A tumour between the optic nerves and anterior to the chiasma will thus apply a stretch to the anterior transversely directed crossing fibres of the chiasma (especially the inferior ones) and bitemporal field defects (at first superior) will develop. As the tumour grows and separation of the nerves and posterior displacement of the chiasma proceeds, more posteriorly placed fibres will in succession be stretched until the most laterally placed, which cross in the posterior chiasma, are involved and a bitemporal hemianopia with splitting of the fixation points results. With a more posteriorly placed tumour which, at an early stage, elevates the chiasma as well as displacing it posteriorly, the more obliquely running and lateral of the crossing fibres will be stretched and homonymous scotomata are then added to the peripheral field defects. With tumours still more posteriorly placed relative to the chiasma as a result of prefixation or other cause, the posteriorly placed crossing macular fibres in the chiasma and medial optic tracts will be stretched and homonymous scotomata will be the sole field defects. Lateral displacement of the optic nerves occasions no increase in tension in the lateral uncrossed fibres of the nerves and chiasma-indeed, it may have the reverse effect. Only when the displacement is, as a result of progressive tumour growth, obstructed by the anterior cerebral or internal carotid artery, will notching of the superolateral fibres occur and loss of vision in the inferior nasal quadrants develop. The inferolateral fibres, relatively protected from the vessels, will be the last to be involved. It is, therefore, believed that field defects from chiasmal lesions frequently depend upon the development of increased tension in particular groups of crossing fibres and not simply on which group of fibres is most closely related to the tumour. Thus a nodule of tumour beneath the lateral chiasma could dis- place the uncrossed fibres, occasioning no increase in tension within them, nor any functional loss. If the nodule were anteriorly placed, a loss of the temporal field on the affected side could be the sole result and would commence with a hemiscotoma, as the most lateral group of crosssing fibres would be first affected; if more posteriorly placed, the crossing fibres from the opposite side would be involved, and a peripheral temporal defect in the field of the opposite eye would be added. At operation in a number of cases the dome of a suprasellar tumour has been seen in such a situation, under one side of the chiasma, spreading the uncrossed and crossing fibres apart from one another. The tension hypothesis may thus not only throw light on the factors responsible for the development of the common form of heteronymous hemianopia but, in addition, possibly that of other less common field defects, the method of production of which is at present ill-understood.

It is possible that this tension hypothesis ha relevance in certain traumatic lesions of the chiasma. At times a bitemporal hemianopi象 develops after a severe blunt head injury, fre quently a frontal one; there may be associate $\phi$ hypothalamic disturbance. In the majority of the reported cases no evidence of a basal skull frako ture which could directly injure the chiasma has been found. It is likely that the lesion is due to traction occasioned by movement of the brain within the skull, and injury to the anatomically delicate and physiologically sensitive area in the median sagittal plane of the chiasma where the crossing fibres interweave. While gross tearing of the chiasma can occur, at exploration no such lesion may be found and the damage would then appear to lie within the pial sheath. The injury to nerve fibres occasioned by such acutely applied tension would be likely to be severe and lead to the permanent bitemporal hemianopia which usually follows. Traquair et al. (1935) favoured a vascular cause for the field defect in these traumatic cases, taking the same view which he held in tumour cases as discussed on an earlier page. It was argued that the chiasma is supplied by a $\frac{D}{O}$ rich network of blood vessels derived from the internal carotid arteries and their branches. The $N$ vessels are firmly attached to the skull base and $N$ movement of the brain and chiasma relative to it N might result in these vessels being torn. It can be 
argued against this vascular hypothesis that the small chiasmal vessels referred to are far removed from the attachment of the internal carotid artery to the skull base and that any stress resulting from brain movement would fall on the major vessel itself. Moreover, the chiasmal vessels lie within the pia mater and arachnoid mater, will move with the structures they supply, and, being extremely tortuous (Dawson, 1958), will be protected from injury by longitudinal stress. Traquair stated that the obvious common factor shared by the traumatic and 'compression' cases was the vascular supply of the chiasma. The anatomy of the chiasma itself is another common factor, however. The chiasma is anchored to the optic canals by the crossed fibres of the optic nerves interweaving in the median sagittal plane. Any posterior displacement of the chiasma is likely to injure these fibres by the marked increase in tension which will develop in them with even minimal chiasmal movement.

It is thus believed that the hypothesis which has been discussed explains a number of the puzzling features of heteronymous hemianopia. In the first place the uncrossed fibres of the optic nerve and chiasma, having no firm posterior attachment, are relatively lax. They can therefore be displaced without any tension developing in them and without any consequent field defect. Only when this displacement is impeded by the internal carotid or anterior cerebral artery will compression of the lateral fibres develop and a nasal field loss result. Secondly, the crossing fibres in the optic nerves are by comparison resistant to displacement because of their interweaving with the crossed fibres from the opposite nerve in the median plane of the chiasma. Displacement laterally of the nerves or posteriorly of the chiasma, whether produced acutely by head injury or gradually by a tumour mass, applies tension to the crossing fibres and the temporal visual field loss develops on a readily understandable anatomical basis. This tension will develop in the crossing fibres in the median plane of the chiasma in spite of variations in the exact size, shape and position of the tumour dome.

It is a pleasure to express my debt to those who have helped in the course of this work. Dr. R. Cureton and Dr. A. G. Stansfeld kindly provided me with excellent facilities in the Department of Histo- pathology of the hospital. In the Department of Medical Illustration skilled photography was always available. The work of Miss Susan Hales was of the highest value, as the published drawings reveal.

\section{REFERENCES}

Bull, J. (1956). The normal variations in the position of the optic recess of the third ventricle. Acta Radiologica, 46, 72-80.

Dawson, B. H. (1958). The blood vessels of the human optic chiasma and their relation to those of the hypophysis and hypothalamus. Brain, 81, 207-217.

Duke-Elder, Sir S., and Scott, G. I. (1971). Neuro-ophthalmology. In System of Ophthalmology. Vol. 12, p. 292. Kimpton: London.

Fisher, J. H. (1913). Discussion on disease of the pituitary body. Proceedings of the Royal Society of Medicine, 6, Liii-LXViii.

Fleischer, B. (1914). Zur Pathologie und Therapie der Hypophysistumoren. Klinische Monatsblätter für Augenheilkunde, 52, 625-653.

Gamble, H. J. (1964). Comparative electron-microscopic observations on the connective tissues of a peripheral nerve and a spinal nerve root in the rat. Journal of Anatomy, 98, 17-25.

Henschen, S. E. (1890 and 1892). Klinische und anatomische Beiträge zur Pathologie des Gehirns. Pts 1 and 2. Almqvist: Upsala.

Kupfer, C., Chumbley, L., and Downer, J. de C. (1967). Quantitative histology of optic nerve, optic tract and lateral geniculate nucleus of man. Journal of Anatomy, 101, 393401.

O'Connell, J. E. A. (1936). The intraneural plexus and its significance. Journal of Anatomy, 70, 468-497.

O'Connell, J. E. A., and du Boulay, E. P. G. H. (1973). Binasal hemianopia. Journal of Neurology, Neurosurgery, and Psychiatry, 36, 697-709.

Polyak, S. L. (1957). The Vertebrate Visual System, pp. 293 and 323. University of Chicago Press: Chicago.

Schaeffer, J. P. (1924). Some points in the regional anatomy of the optic pathway, with especial reference to tumors of the hypophysis cerebri and resulting ocular changes. Anatomical Record, 28, 243-279.

Scott, G. I. (1957). (Ed.). Traquair's Clinical Perimetry, 7th edn, pp. 70 and 208. Kimpton: London.

Sunderland, S., and Bradley, K. C. (1961a). Stress-strain phenomena in human peripheral nerve trunks. Brain, 84, 102-119.

Sunderland, S., and Bradley, K. L. (1961b). Stress-strain phenomena in human spinal nerve roots. Brain, 84, 120 124.

Traquair, H. M. (1917). Bitemporal hemiopia: the later stages and the special features of the scotoma. British Journal of Ophthalmology, 1, 216-239, 281-294, and 337352.

Traquair, H. M., Dott, N. M., and Russell, W. R. (1935). Traumatic lesions of the optic chiasma. Brain, 58, 398-411.

Walker, C. B., and Cushing, H. (1918). Chiasmal lesions, with especial reference to homonymous hemianopsia with hypophyseal tumor. Archives of Ophthalmology, 47, 119145.

Whitnall, S. E. (1932). The Anatomy of the Human Orbit. 2nd edn., p. 382. Oxford University Press: London.

Wilbrand, H., and Sänger, A. (1904). Die Neurologie des Auges. Bergmann: Wiesbaden. Vol. 3, Abt 1, pp. 98-etc.

Zander, R. (1896). Beiträge zur Kenntnis der mittleren Schädelgrube mit besonderer Berücksichtigung der Lage des Chiasma opticum. Anatomischer Anzeiger, 12, 457-467. 